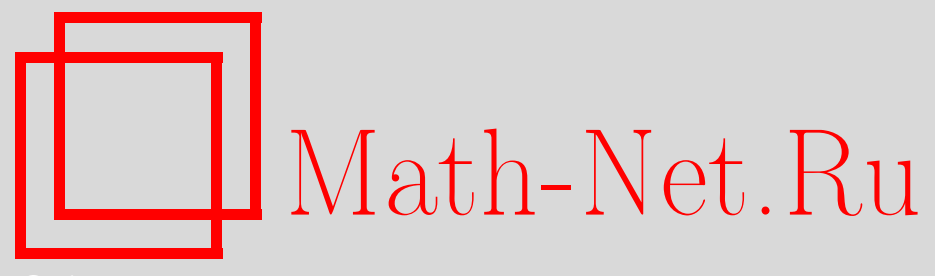

А. М. Локощенко, Л. В. Фомин, Ю. Г. Басалов, В. С. Агабабян, Моделирование ползучести металлов при нестационарном сложном напряженном состоянии, Вестн. Сам. гос. техн. ун-та. Сер. Физ.-мат. науки, 2019, номер 1, 69-89

DOI: https://doi.org/10.14498/vsgtu1668

Использование Общероссийского математического портала MathNet.Ru подразумевает, что вы прочитали и согласны с пользовательским соглашением

http://www.mathnet.ru/rus/agreement

Параметры загрузки:

IP: 54.237 .59 .107

26 апреля 2023 г., $17: 37: 35$

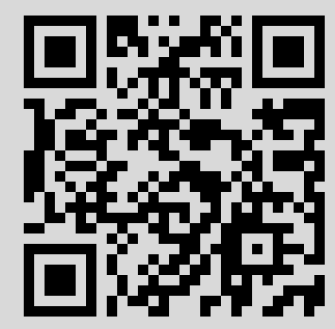


Вестн. Сам. гос. техн. ун-та. Сер. Физ.-мат. науки. 2019. Т. 23, № 1. С. $69-89$ ISSN: 2310-7081 (online), 1991-8615 (print)

УДК 539.376

\title{
Моделирование ползучести металлов при нестационарном сложном напряженном состоянии
}

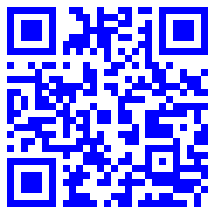

\author{
А. М. Локощенко ${ }^{1}$, Л. В. Фомин ${ }^{1}$, \\ Ю. Г. Басалов ${ }^{1}$, В. С. Агабабян \\ 1 Московский государственный университет имени М. В. Ломоносова, \\ Научно-исследовательский институт механики, \\ Россия, 119192, Москва, Мичуринский проспект, 1. \\ 2 Московский государственный университет имени М. В. Ломоносова, \\ Механико-математический факультет, \\ Россия, 119991, Москва, Ленинские горы, 1.
}

\section{Аннотация}

Рассмотрено моделирование результатов испытаний металлов в условиях ползучести при нестационарном сложном напряженном состоянии. В качестве примера рассмотрены экспериментальные данные, полученные группой японских ученых при испытаниях трубчатых образцов из нержавеющей стали при температуре $650^{\circ} \mathrm{C}$. В приведенной статье представлены результаты испытаний при четырех различных программах нагружения. Эти программы нагружения представляют собой различные комбинации кусочно-постоянных зависимостей касательного и нормального напряжений от времени. Проведено моделирование представленных данных с помощью теории упрочнения и теории течения, две используемые материальные константы определяются из условия минимального относительного интегрального расхождения экспериментальных и теоретических кривых ползучести. Проведено сопоставление результатов моделирования с результатами моделирования этих тех же экспериментальных данных, полученных другими исследователями с использованием других теорий. В этих других теориях использовано большое количество характеристик материала: от трех до девяти констант и дополнительно - одной материальной функции. Показано преимущество рассмотренных авторами данной статьи теории упрочнения и теории течения всего с двумя материальными константами в каждой по сравнению с другими использованными теориями.

\section{Научная статья}

(2)(7) Контент публикуется на условиях лицензии Creative Commons Attribution 4.0 International (https://creativecommons.org/licenses/by/4.0/deed.ru)

\section{Образец для цитирования}

Локощенко А. М., Фомин Л. В., Басалов Ю. Г., Агабабян В. С. Моделирование ползучести металлов при нестационарном сложном напряженном состоянии // Becmн. Cам. гос. техн. ун-та. Сер. Физ.-мат. науки, 2019. Т. 23, № 1. С. 69-89. doi:10.14498/vsgtu1668.

\section{Сведения об авторах}

Александр Михайлович Локощенко (1) https://orcid.org/0000-0002-5462-6055 доктор физико-математических наук, профессор; заведующий лабораторией; лаб. ползучести и длительной прочности; e-mail: loko@imec.msu.ru

Леонид Викторович Фомин (10) https://orcid.org/0000-0002-9075-5049

кандидат физико-математических наук; старший научный сотрудник; лаб. ползучести и длительной прочности; e-mail: fleonid1975@mail.ru

Юрий Генрихович Басалов; ведущий инженер; лаб. ползучести и длительной прочности; e-mail: basalov@yandex .ru

Вардан Самвелович Агабабян; студент; каф. газовой и волновой динамики 
Ключевые слова: ползучесть металлов, эксперименты, нестационарное сложное напряженное состояние, моделирование, теория упрочнения, теория течения.

Получение: 28 декабря 2018 г. / Исправление: 26 февраля 2019 г. Принятие: 4 марта 2019 г. / Публикация онлайн: 5 марта 2019 г.

Введение. Подавляющая часть экспериментальных и теоретических исследований ползучести металлов - исследования поведения металлов при одноосном растяжении. Однако на практике реальные конструкции при высоких температурах находятся в условиях сложного напряженного состояния. Проведение испытаний на высокотемпературную ползучесть связано с большими трудностями, поэтому такие испытания, тем более при переменных напряжениях, представляют большой интерес. Результаты испытаний тонкостенных трубчатых образцов при нестационарном сложном напряженном состоянии приведены, в частности, в [1-4]. Моделирование длительной прочности в этих условиях проведено А. М. Локощенко в $[5,6]$, при этом было использовано развитие кинетической теории Ю. Н. Работнова [7] при учете векторного параметра поврежденности.

В данной статье рассматриваются экспериментальные данные, полученные группой японских исследователей [8]. Эти данные получены на трубчатых образцах из нержавеющей стали 304, испытанных на ползучесть при температуре $650^{\circ} \mathrm{C}$ в условиях кусочно-постоянных зависимостей касательного и нормального напряжений от времени. Анализ этих экспериментальных данных и описание результатов с помощью различных моделей представляют большой интерес.

1. Результаты испытаний. В работе [8] приведены результаты испытаний трубчатых образцов из нержавеющей стали 304 с наружным диаметром $21 \pm 0.5$ мм, толщиной стенки $1 \pm 0.05$ мм и рабочей длиной 98 мм. Перед механической обработкой материал подвергался термической обработке при $1100{ }^{\circ} \mathrm{C}$, после которой были получены следующие механические характеристики при комнатной температуре: предел текучести 274.6 МПа, предел прочности 637.4 МПа и твердость по Бринеллю 174. В процессе испытаний температура испытаний измерялась тремя платино-платинородиевыми термопарами, приваренными точечной сваркой в трех точках вдоль рабочей длины образца. Отклонение температуры испытаний от $650{ }^{\circ} \mathrm{C}$ составляло менее $0.5^{\circ} \mathrm{C}$ по времени и $1.5^{\circ} \mathrm{C}$ по длине образцов. Перед испытаниями образцы выдерживались при температуре испытаний $650{ }^{\circ} \mathrm{C}$ около 22 часов.

В данных испытаниях сложное напряженное состояние создавалось кусочно-постоянными зависимостями нормального $\sigma$ и касательного $\tau$ напряжений от времени $t$ в течение 80 часов, скачкообразное изменение величин $\sigma$ и $\tau$ осуществлялось при $t=k t_{0}$, где $k=0,1, \ldots, 10, t_{0}=8$ часов, при этом испытания представляют собой последовательные 5 циклов по $2 t_{0}=$ $=16$ часов (рис. 1-4). В процессе всех испытаний интенсивность напряжений $\sigma_{u}=\sqrt{\sigma^{2}+3 \tau^{2}}$ сохраняла постоянное значение: $\sigma_{u}=\sigma_{0}=137.3$ МПа, этот уровень напряжений почти равен пределу текучести при $650{ }^{\circ} \mathrm{C}$. 
В работе [8] представлены результаты зависимостей осевой $p_{z}(t)$ и сдвиговой $\gamma^{c}(t)$ деформаций ползучести от времени $t$, при этом интенсивность деформаций ползучести вычисляется по формуле

$$
p_{u}(t)=\sqrt{\left(p_{z}(t)\right)^{2}+(\Gamma(t))^{2}}, \quad \Gamma(t)=\frac{\gamma^{c}(t)}{\sqrt{3}} .
$$

На рис. 1-4 приведены экспериментальные зависимости величин $\Gamma(t)$ и $p_{z}(t)$ при четырех различных программах нагружения.

В состоянии разгрузки осевая и сдвиговая деформации ползучести практически сохраняют свои значения. В четвертой программе нагружения образцы находятся только при знакопеременном кручении (осевое напряжение отсутствует), при этом кривые сдвиговой деформации на всех циклах нагружения практически совпадают. Отсюда следует, что поврежденность материала, обычно накапливающуюся при испытаниях такого типа, в данном случае можно не учитывать.

2. Моделирование полученных результатов в [8-10]. В работе [8], кроме экспериментальных кривых ползучести, приведены теоретические кривые, соответствующие различным теориям (модели 1-4).

Стандартная теория старения (модель № 1), называемая в статье [8] теорией деформационного упрочнения, рассматривается в виде обобщения уравнения Бейли-Нортона на случай сложного напряженного состояния.

В модифицированной теории старения (модель № 2) дополнительно учитывается изменение знака касательного напряжения.

В теории кинематического упрочнения (модель № 3) используется подход Н. Н. Малинина и Г. М. Хажинского [9] с введением дополнительных гипотез.

В теории смешанного упрочнения (модель № 4) используются основные понятия физики твердого тела (теория дислокаций и др.).

При этом в первой и второй указанных моделях использованы по 3 материальные константы, в третьей модели - 5 материальных констант и 1 материальная функция, в четвертой модели - 6 констант и 1 материальная функция.

В работе [10] приведены результаты моделирования экспериментальных данных [8] с помощью варианта уравнений нестационарной ползучести (модель № 5), предложенного Ю. Г. Коротких и развитого в работах его учеников. В этой модели принимается, что компоненты тензора деформаций являются суммами трех составляющих: упругих деформаций, пластических деформаций и деформаций ползучести. В модели учитываются уровень температуры $T$, зависимости модуля сдвига, объемного модуля упругости и коэффициента температурного расширения от $T$, упругое изменение объема тела, поврежденность материала и другие параметры. В модели № 5 использованы 9 материальных констант и одна материальная функция. В [11] дополнительно рассмотрены два частных варианта рассматриваемой модели.

\section{3. Моделирование результатов испытаний [8] с помощью теории} упрочнения (модель № 6). В данном пункте приведены результаты моделирования экспериментальных данных [8] с помощью теории упрочнения при четырех программах нагружения. 

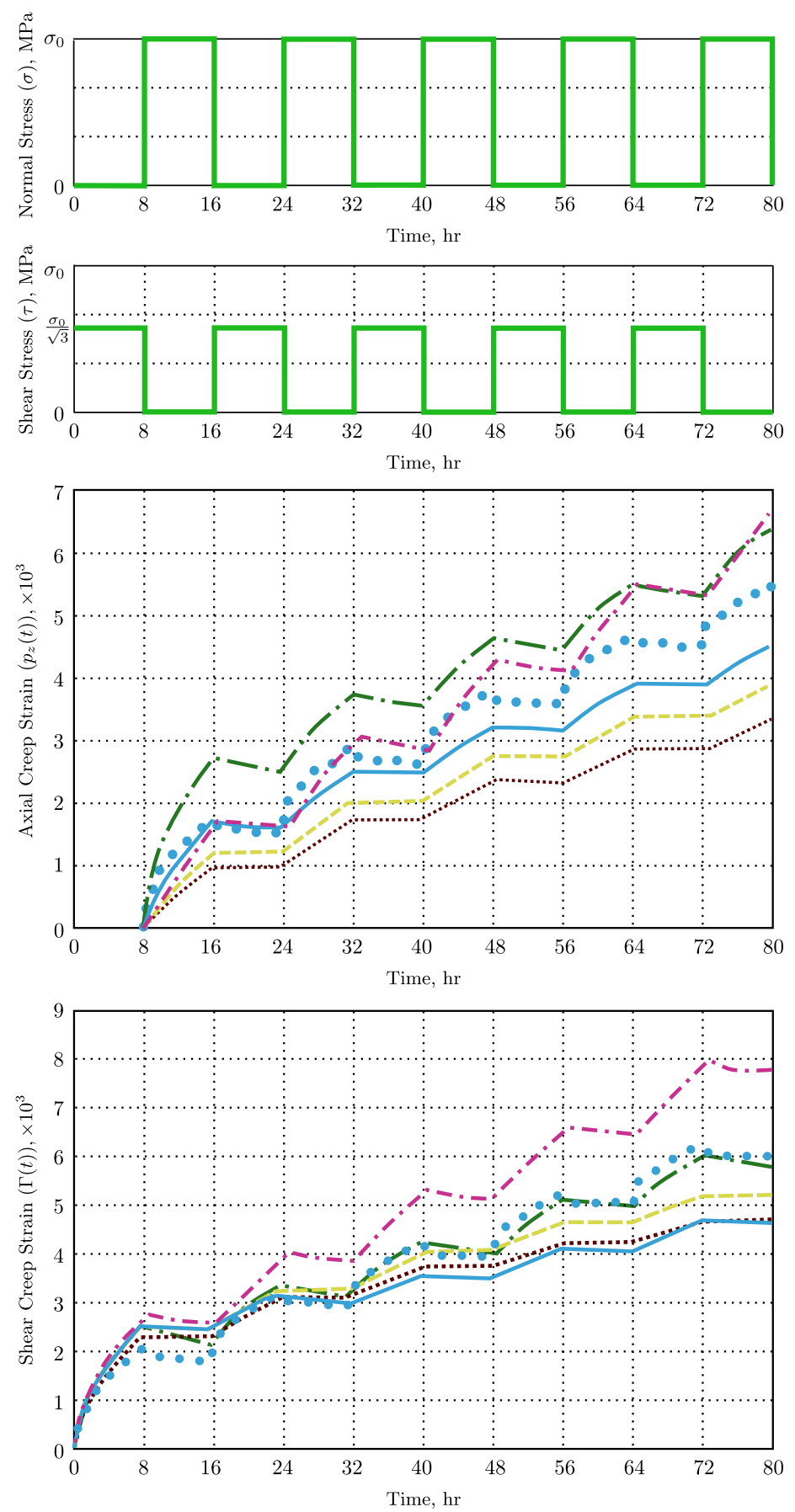

- Experimental data $[8]$

..... Standard theory of aging [8] (model no. 1)

- - Modified theory of aging [8] (model no. 2)

- Theory of kinematic hardening $[8,9]$ (model no. 3)

- Theory of mixed hardening [8] (model no. 4)

- Theory of nonstationary creep [10] (model no. 5)

Рис. 1. (онлайн в цвете) Первая программа нагружения, экспериментальные данные и результаты расчета по различным моделям [Figure 1. (color online) The first loading program, 

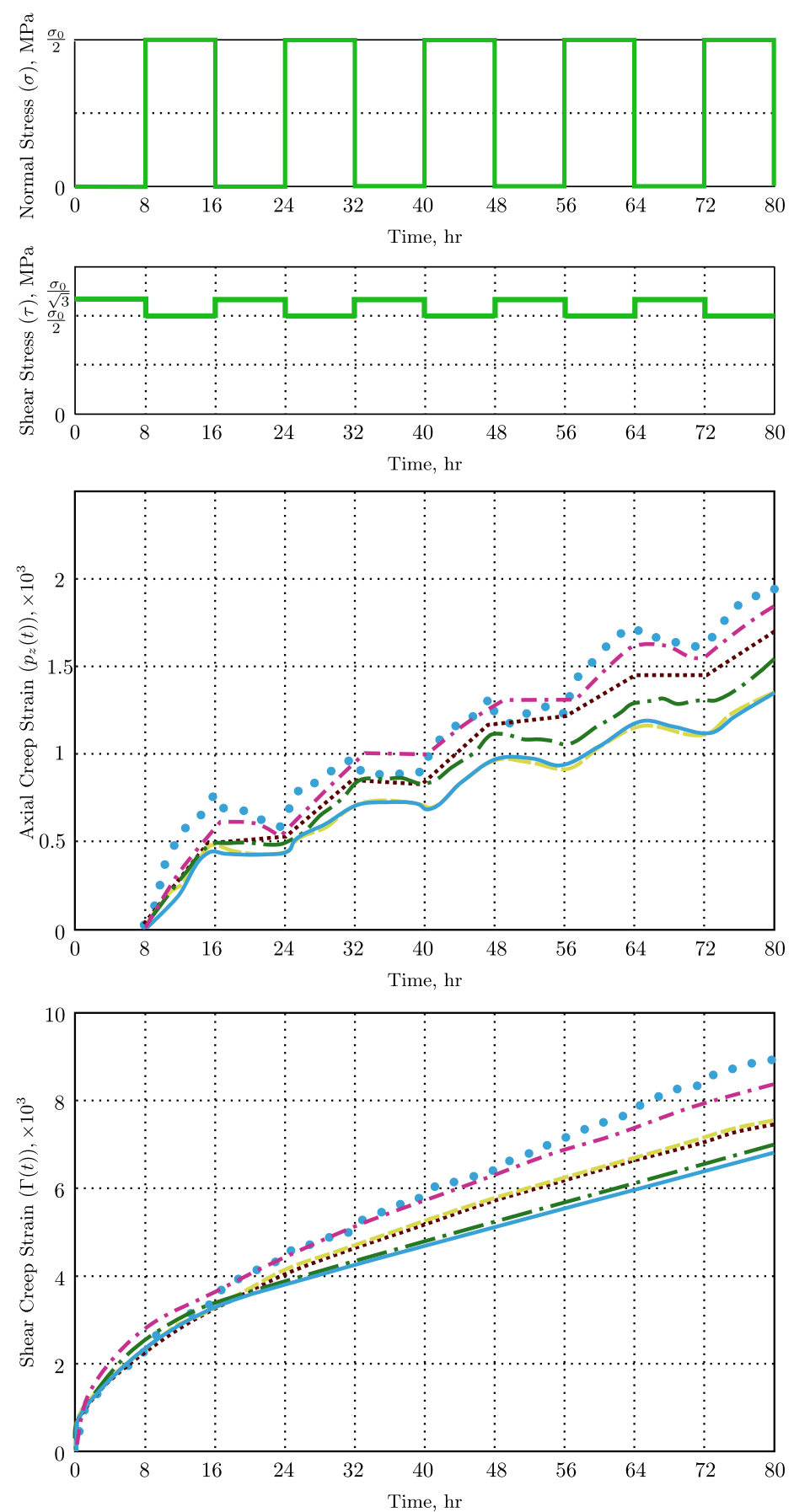

- Experimental data $[8]$

..... Standard theory of aging [8] (model no. 1)

- Modified theory of aging [8] (model no. 2)

- Theory of kinematic hardening $[8,9]$ (model no. 3)

- Theory of mixed hardening [8] (model no. 4)

- " Theory of nonstationary creep [10] (model no. 5)

Рис. 2. (онлайн в цвете) Вторая программа нагружения, экспериментальные данные и результаты расчета по различным моделям [Figure 2. (color online) The second loading program, experimental data, and calculation results for various models] 

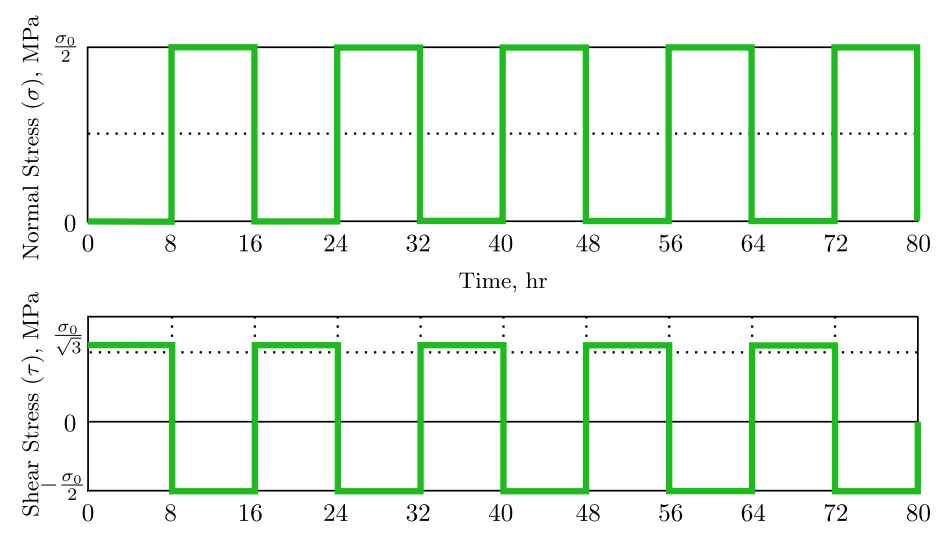

Time, hr
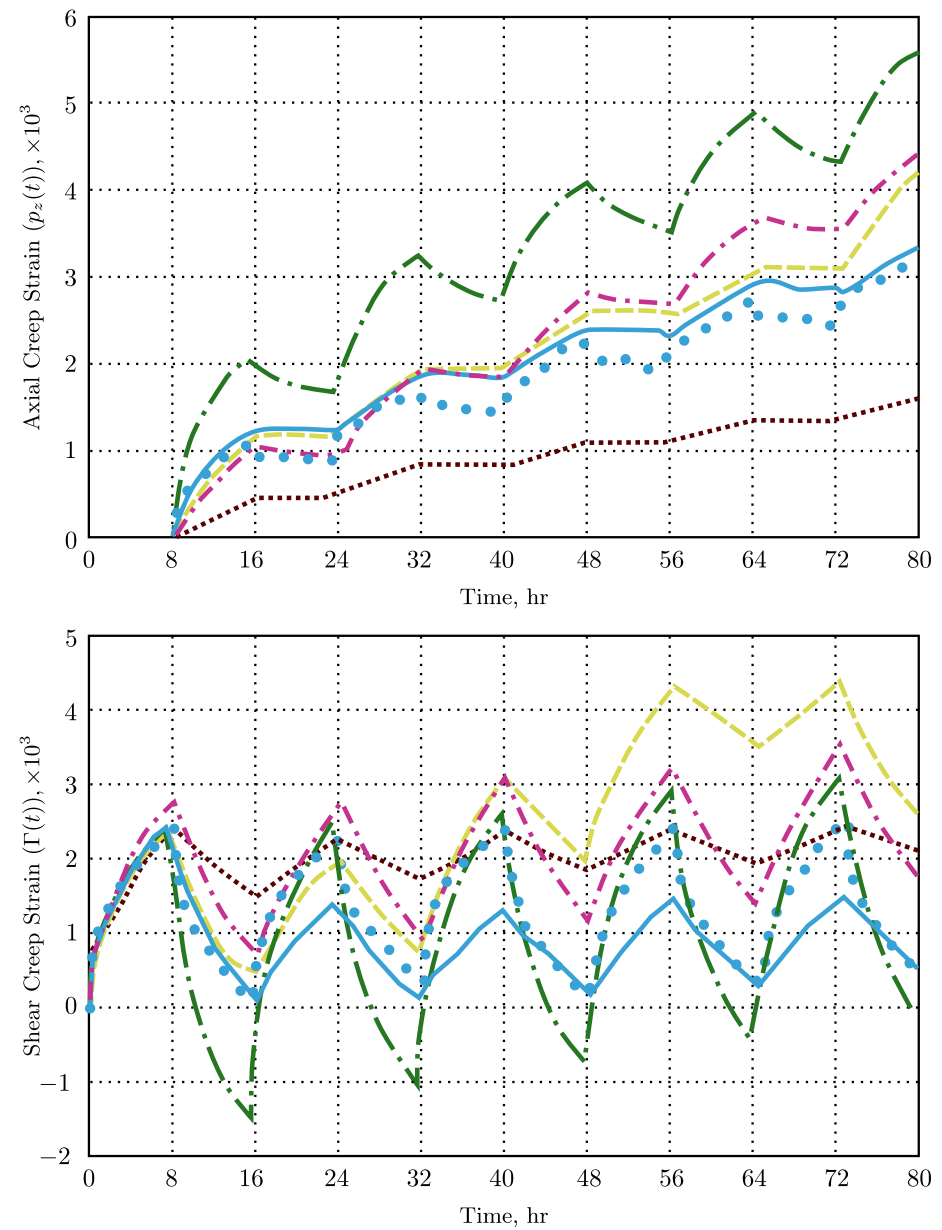

- Experimental data [8]

..... Standard theory of aging [8] (model no. 1)

- Modified theory of aging [8] (model no. 2)

- Theory of kinematic hardening $[8,9]$ (model no. 3)

- Theory of mixed hardening [8] (model no. 4)

- . Theory of nonstationary creep [10] (model no. 5)

Рис. 3. (онлайн в цвете) Третья программа нагружения, экспериментальные данные и результаты расчета по различным моделям [Figure 3. (color online) The third loading program, experimental data, and calculation results for various models] 

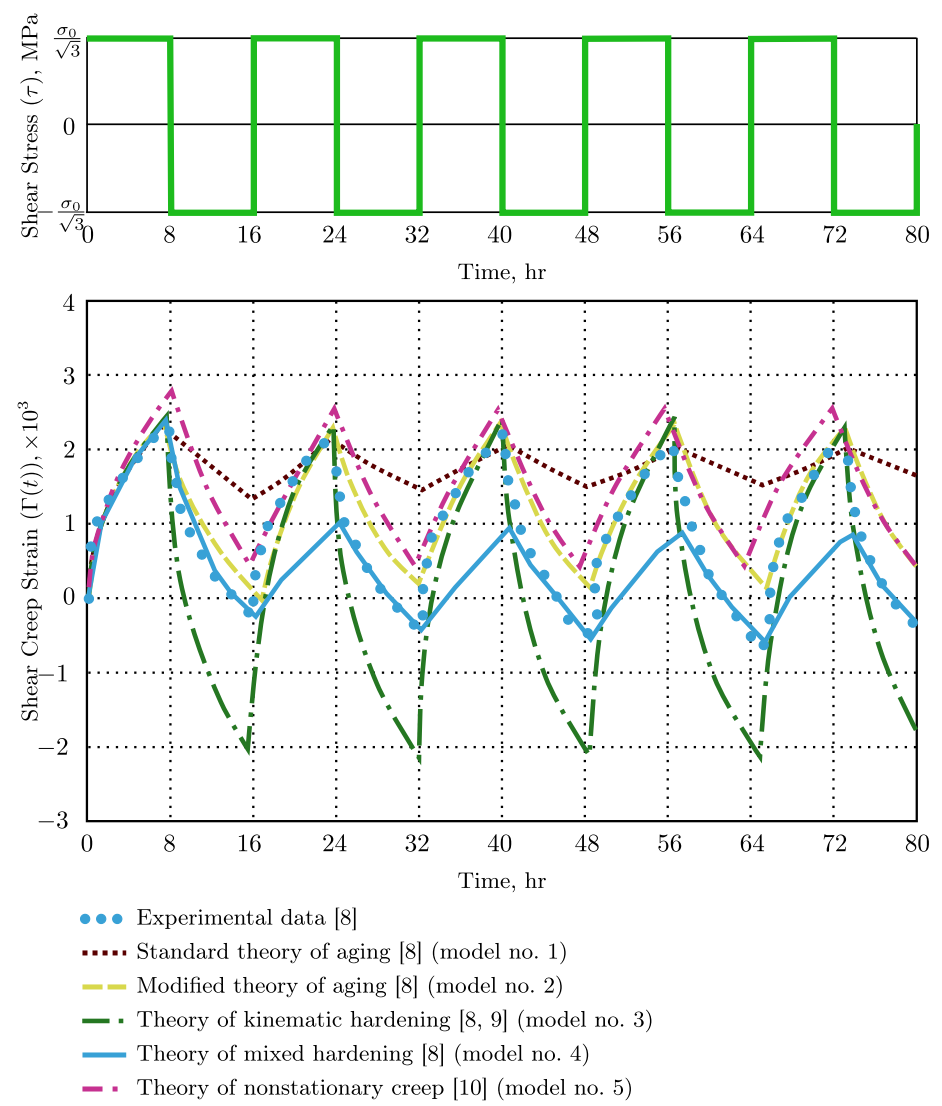

Рис. 4. (онлайн в цвете) Четвертая программа нагружения, экспериментальные данные и результаты расчета по различным моделям [Figure 4. (color online) The fourth loading program, experimental data, and calculation results for various models]

В первой программе нагружения в каждый момент времени образцы находились либо при одностороннем кручении, либо при одноосном растяжении (см. рис. 1).

Во второй и третьей программах касательное напряжение $\tau$ отлично от нуля при любом значении времени $t$, в то время как зависимость нормального напряжения $\sigma$ от времени принимает попеременно нулевое и положительное значения (см. рис. 2 и 3 соответственно).

В четвертой программе нагружения в каждый момент времени образцы находились только при прямом и обратном кручении (см. рис. 4).

В работе [8] указано, что скорость нагружения и разгрузки составила $49 \mathrm{MПа/мин,} \mathrm{это} \mathrm{означает,} \mathrm{что} \mathrm{длительность} \mathrm{нагружения} \mathrm{и} \mathrm{разгрузки} \mathrm{со-}$ ставляла 2.8 мин, отношение этого времени к продолжительности каждого этапа ползучести (8 часов) составляло всего $0.58 \%$. Поэтому при моделировании экспериментальных данных принимается, что процессы нагружения и разгрузки происходили мгновенно. Сдвиговые и продольные деформации ползучести при $t=0$ по определению равны нулю: $\Gamma(0)=0, p_{z}(0)=0$.

В 1948 г. Ю. Н. Работнов [12] предложил простое уравнение неустановившейся ползучести при осевом растяжении:

$$
p^{\alpha} \dot{p}=f(\sigma), \quad 0<\alpha<1,
$$


которое естественным образом обобщается на сложное напряженное состояние; точкой здесь и далее обозначается дифференцирование по времени.

Примем гипотезу, согласно которой компоненты девиаторов напряжений $s_{i j}$ и скоростей деформаций ползучести несжимаемого материала $p_{i j}$ пропорциональны [13]:

$$
\dot{p_{i j}}=\frac{3}{2} \frac{f\left(\sigma_{u}\right)}{p_{u}^{\alpha}} \frac{s_{i j}}{\sigma_{u}}=\frac{3}{2} \frac{f\left(\sigma_{0}\right)}{p_{u}^{\alpha}} \frac{s_{i j}}{\sigma_{0}}=\frac{3}{2} \frac{D_{1}}{p_{u}^{\alpha}} \frac{s_{i j}}{\sigma_{0}}=\frac{3}{2} \frac{D_{1}}{\left(p_{z}^{2}+\Gamma^{2}\right)^{\alpha / 2}} \frac{s_{i j}}{\sigma_{0}}
$$

где $\sigma_{u}=\sigma_{0}=137.3 \mathrm{MПа;} D_{1}=f\left(\sigma_{0}\right)=A \sigma_{0}^{n}(A, n-$ параметры $)$.

Из уравнений (1) следует, что как при кручении, так и при растяжении при полной разгрузке соответствующие деформации ползучести сохраняют постоянное значение, накопленное в конце предыдущего этапа перед разгрузкой. В рассматриваемых испытаниях деформация ползучести не только после полной разгрузки, но и после частичной разгрузки составляет всего несколько процентов по отношению к накопленному перед разгрузкой уровню деформации ползучести, поэтому в этом случае уравнение (1) можно заменить на следующее:

$$
p_{i j}=0 \text {. }
$$

В принципе, можно рассмотреть более точное моделирование процесса ползучести после разгрузки с учетом явления последействия с помощью кинетической теории Ю. Н. Работнова, которое в случае одноосного растяжения описывается следующей системой уравнений:

$$
\begin{cases}\dot{p}=A(\sigma-q)^{n}, & p(0)=0, \\ \dot{q}=B \sigma-D q, & q(0)=0\end{cases}
$$

где $q(t)$ - кинетический параметр. В [13] показано, что при полной разгрузке в момент времени $t_{1}$ деформация ползучести при $t>t_{1}$ постепенно уменьшается до некоторого предельного значения. Однако, так как в рассматриваемых испытаниях деформация ползучести в процессе разгрузки уменьшается очень незначительно, в данном случае такое уточнение предполагается излишним.

3.1. Первая программа нагружения. При анализе процесса ползучести выделим 10 временных отрезков (этапов нагружения): $0<t<t_{0}$, $t_{0}<t<2 t_{0}, 2 t_{0}<t<3 t_{0}, \ldots, 9 t_{0}<t<10 t_{0}$ и присвоим им номера $k=1,2,3, \ldots, 10$.

Согласно рис. 1, компоненты тензоров напряжений на нечетном и четном интервалах действия напряжений имеют следующие значения:

$$
\begin{array}{llll}
k=1,3,5,7,9: & \tau(t)=\frac{\sigma_{0}}{\sqrt{3}}, & \sigma(t)=0 ; \\
k=2,4,6,8,10: & \tau(t)=0, & \sigma(t)=\sigma_{0} .
\end{array}
$$

При положительных значениях $\tau(t)=\sigma_{0} / \sqrt{3}$ и $\sigma(t)=\sigma_{0}$, согласно $(1)$, имеем

$$
\frac{d p_{z \theta}}{d t}=\frac{1}{2} \frac{d \gamma^{c}}{d t}=\frac{\sqrt{3}}{2} \cdot \frac{1}{\sqrt{3}} \frac{d \gamma^{c}}{d t}=\frac{\sqrt{3}}{2} \frac{d \Gamma}{d t}=\frac{3}{2} \frac{D_{1}}{\left(p_{z}^{2}+\Gamma^{2}\right)^{\alpha / 2}}\left(\frac{1}{\sigma_{0}} \cdot \frac{\sigma_{0}}{\sqrt{3}}\right)
$$




$$
\frac{d p_{z}}{d t}=\frac{3}{2} \frac{D_{1}}{\left(p_{z}^{2}+\Gamma^{2}\right)^{\alpha / 2}}\left(\frac{1}{\sigma_{0}} \cdot \frac{2}{3} \sigma_{0}\right)
$$

Здесь и далее введена цилиндрическая система координат $(r, \theta, z)$, ось $z$ которой направлена вдоль продольной оси симметрии цилиндрического трубчатого образца, $r$ - координата вдоль радиуса, $\theta$ - угол радиус-вектора $r$ в рассматриваемой точке. При этом $p_{z z} \equiv p_{z}$ - нормальная компонента тензора деформаций ползучести, а $p_{z \theta}$ - касательная компонента тензора деформаций ползучести.

Из последних соотношений с учетом (2), (3) получаем

$$
\begin{gathered}
\left.\Gamma\right|_{t=0}=0, \quad \frac{d \Gamma}{d t}=\frac{D_{1}}{\left(p_{z}^{2}+\Gamma^{2}\right)^{\alpha / 2}} \text { при } \tau=\frac{\sigma_{0}}{\sqrt{3}}, \quad \frac{d \Gamma}{d t}=0 \text { при } \tau=0 ; \\
\left.p_{z}\right|_{t=0}=0, \quad \frac{d p_{z}}{d t}=\frac{D_{1}}{\left(p_{z}^{2}+\Gamma^{2}\right)^{\alpha / 2}} \text { при } \sigma=\sigma_{0}, \quad \frac{d p_{z}}{d t}=0 \text { при } \sigma=0 .
\end{gathered}
$$

С помощью (4), (5) вычислим значения компонент тензора деформаций ползучести при значениях времен, соответствующих окончаниям последовательных этапов нагружения. Значения сдвиговой деформации $\Gamma(t)$ в конце $k$ того отрезка обозначим через $\Gamma_{k}$, а значения осевой деформации $p_{z}(t)$ в конце $k$-того отрезка времени $-P_{k}$. Тогда

1) если $k=1$, то $0<t<t_{0} ; \sigma(t)=0, p_{z}(t)=0, p_{z}\left(t_{0}\right)=P_{1}=0 ; \tau(t)=\frac{\sigma_{0}}{\sqrt{3}}$,

$$
\begin{aligned}
& \frac{d \Gamma}{d t}=\frac{D_{1}}{\Gamma^{\alpha}}, \Gamma(0)=0, \Gamma_{1}=\Gamma\left(t_{0}\right) ; \int_{0}^{\Gamma_{1}} \Gamma^{\alpha} d \Gamma=\left.\frac{\Gamma^{(\alpha+1)}}{(\alpha+1)}\right|_{0} ^{\Gamma_{1}}=\frac{\Gamma_{1}^{(\alpha+1)}}{(\alpha+1)}= \\
& =D_{1} t_{0}, \Gamma_{1}=\left[(\alpha+1) D_{1} t_{0}\right]^{\frac{1}{(\alpha+1)}} ;
\end{aligned}
$$

2) если $k=2$, то $t_{0}<t<2 t_{0} ; \tau(t)=0, \Gamma(t)=\Gamma_{1}, \frac{d \Gamma}{d t}=0, \Gamma\left(2 t_{0}\right)=\Gamma_{2}=\Gamma_{1}$; $\sigma(t)=\sigma_{0}, \frac{d p_{z}}{d t}=\frac{D_{1}}{\left(p_{z}^{2}+\Gamma_{1}^{2}\right)^{\alpha / 2}}, p_{z}\left(t_{0}\right)=P_{1}=0, p_{z}\left(2 t_{0}\right)=P_{2}$

3) если $k=3,5,7,9$, то $(k-1) t_{0}<t<k t_{0} ; \sigma(t)=0, p_{k}\left(k t_{0}\right)=P_{k}=P_{k-1}$, $\tau(t)=\frac{\sigma_{0}}{\sqrt{3}}, \Gamma\left((k-1) t_{0}\right)=\Gamma_{k-1}, \frac{d \Gamma}{d t}=\frac{D_{1}}{\left(P_{k}^{2}+\Gamma^{2}\right)^{\alpha / 2}}, \Gamma\left(k t_{0}\right)=\Gamma_{k} ;$

4) если $k=4,6,8,10$, то $(k-1) t_{0}<t<k t_{0}$;

$$
\begin{aligned}
& \tau(t)=0, \Gamma\left((k-1) t_{0}\right)=\Gamma_{k-1}, \frac{d \Gamma}{d t}=0, \Gamma_{k}=\Gamma_{k-1} ; \\
& \sigma(t)=\sigma_{0}, p_{z}\left((k-1) t_{0}\right)=P_{k-1}, \frac{d p_{z}}{d t}=\frac{D_{1}}{\left(p_{z}^{2}+\Gamma_{k}^{2}\right)^{\alpha / 2}}, P_{z}\left(k t_{0}\right)=P_{k} .
\end{aligned}
$$

Теоретические кривые, соответствующие модели № 6 , для первой программы нагружения приведены на рис. 5 .

3.2. Вторая программа нагружения. Согласно рис. 2, компоненты тензоров напряжений при нечетном и четном интервалах времени имеют следующие значения:

$$
\begin{array}{lll}
k=1,3,5,7,9: & \tau(t)=\frac{\sigma_{0}}{\sqrt{3}}, & \sigma(t)=0 \\
k=2,4,6,8,10: & \tau(t)=\frac{\sigma_{0}}{2}, & \sigma(t)=\frac{\sigma_{0}}{2}
\end{array}
$$



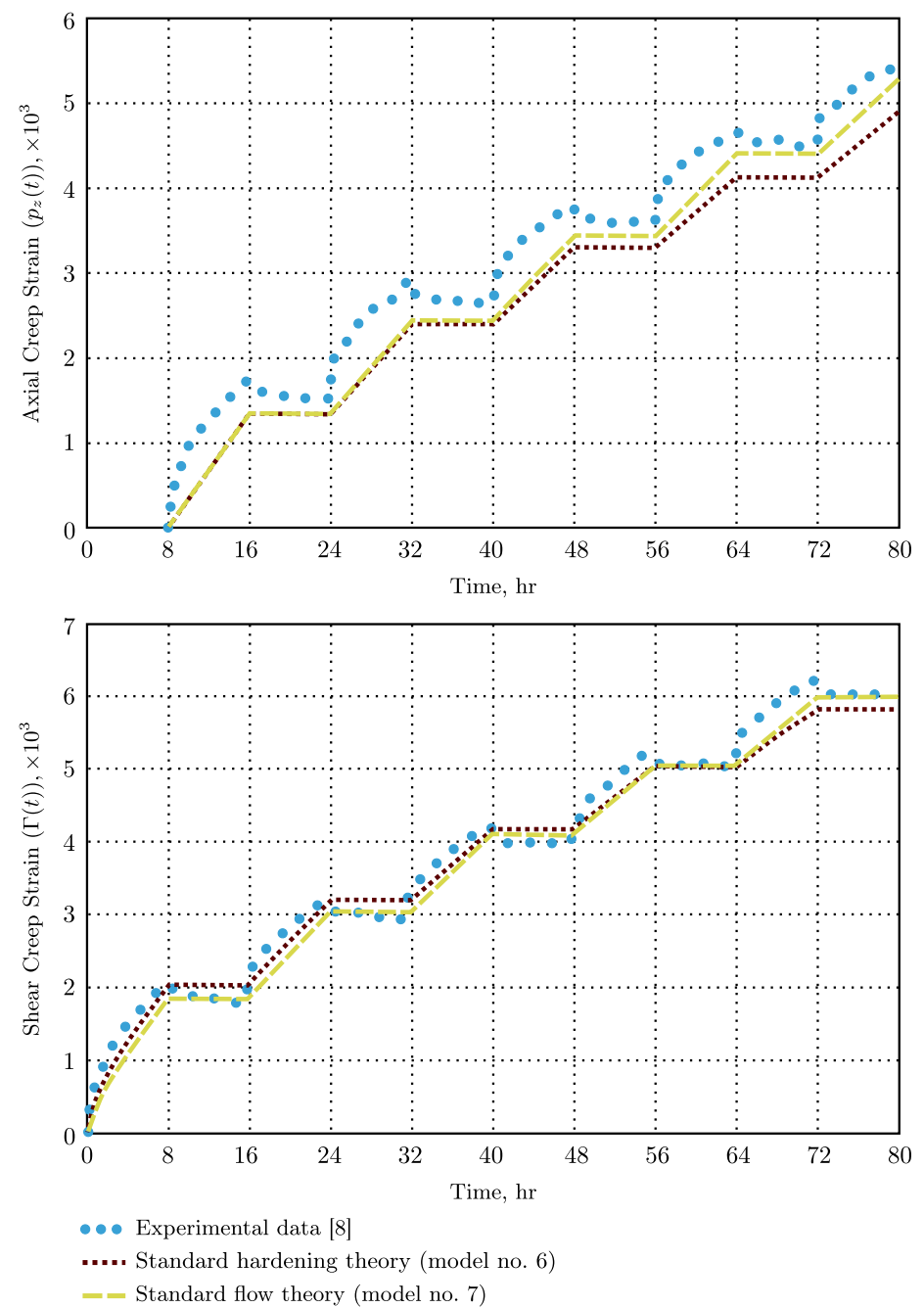

Рис. 5. (онлайн в цвете) Экспериментальные данные и результаты расчета по различным моделям для первой программы нагружения [Figure 5. (color online) Experimental data, and calculation results for various models for the first loading program]

Из (1), (2) аналогично (4), (5) с учетом (6) получаем

$$
\begin{gathered}
\frac{d \Gamma}{d t}=\frac{D_{1}}{\left(p_{k}^{2}+\Gamma^{2}\right)^{\alpha / 2}} \text { при } \tau=\frac{\sigma_{0}}{\sqrt{3}}, \quad \frac{d \Gamma}{d t}=\frac{\sqrt{3}}{2} \frac{D_{1}}{\left(p_{k}^{2}+\Gamma^{2}\right)^{\alpha / 2}} \text { при } \tau=\frac{\sigma_{0}}{2} ; \\
\frac{d p_{z}}{d t}=\frac{D_{1}}{2\left(p_{z}^{2}+\Gamma^{2}\right)^{\alpha / 2}} \text { при } \sigma(t)=\frac{\sigma_{0}}{2}, \quad \frac{d p_{z}}{d t}=0 \text { при } \sigma(t)=0 .
\end{gathered}
$$

Далее с помощью (7), (8) при учете (6) проведено вычисление компонент тензоров деформаций ползучести в концах различных этапов нагружения:

1) если $k=1$, то $0<t<t_{0} ; \tau(t)=\frac{\sigma_{0}}{\sqrt{3}}, \sigma(t)=0, p_{z}(t)=0, p_{z}\left(t_{0}\right)=$ $=P_{1}=0 ; \frac{d \Gamma}{d t}=\frac{D_{1}}{\left(P_{k}^{2}+\Gamma^{2}\right)^{\alpha / 2}}=\frac{D_{1}}{\Gamma^{\alpha}}, \Gamma(0)=0, \Gamma\left(t_{0}\right)=\Gamma_{1} ; \int_{0}^{\Gamma_{1}} \Gamma^{\alpha} d \Gamma=$ 


$$
=\left.\frac{\Gamma^{(\alpha+1)}}{(\alpha+1)}\right|_{0} ^{\Gamma_{1}}=\frac{\Gamma_{1}^{(\alpha+1)}}{(\alpha+1)}=D_{1} t_{0}, \Gamma\left(t_{0}\right)=\Gamma_{1}=\left[(\alpha+1) D_{1} t_{0}\right]^{\frac{1}{(\alpha+1)}}
$$

2 ) если $k=2$, то $t_{0}<t<2 t_{0} ; \tau(t)=\frac{\sigma_{0}}{2}, \sigma(t)=\frac{\sigma_{0}}{2}$;

$$
\begin{cases}\frac{d \Gamma}{d t}=\frac{\sqrt{3}}{2} \frac{D_{1}}{\left(p_{z}^{2}+\Gamma^{2}\right)^{\alpha / 2}}, & \Gamma\left(t_{0}\right)=\Gamma_{1}, \Gamma\left(2 t_{0}\right)=\Gamma_{2} \\ \frac{d p_{z}}{d t}=\frac{1}{2} \frac{D_{1}}{\left(p_{z}^{2}+\Gamma^{2}\right)^{\alpha / 2}}, & p_{z}\left(t_{0}\right)=0, p_{z}\left(2 t_{0}\right)=P_{2}\end{cases}
$$

3) если $k=3,5,7,9$, то $(k-1) t_{0}<t<k t_{0} ; \tau(t)=\frac{\sigma_{0}}{\sqrt{3}}, \sigma(t)=0, P_{k}=P_{k-1}$; $\frac{d \Gamma}{d t}=\frac{D_{1}}{\left(P_{k}^{2}+\Gamma^{2}\right)^{\alpha / 2}}, \Gamma\left((k-1) t_{0}\right)=\Gamma_{k-1}, \Gamma\left(k t_{0}\right)=\Gamma_{k}$

4) если $k=4,6,8,10$, то $(k-1) t_{0}<t<k t_{0} ; \tau(t)=\frac{\sigma_{0}}{2}, \sigma(t)=\frac{\sigma_{0}}{2}$;

$$
\begin{cases}\frac{d \Gamma}{d t}=\frac{\sqrt{3}}{2} \frac{D_{1}}{\left(p_{z}^{2}+\Gamma^{2}\right)^{\alpha / 2}}, & \Gamma\left((k-1) t_{0}\right)=\Gamma_{k-1}, \Gamma\left(k t_{0}\right)=\Gamma_{k} \\ \frac{d p_{z}}{d t}=\frac{1}{2} \frac{D_{1}}{\left(p_{z}^{2}+\Gamma^{2}\right)^{\alpha / 2}}, & p_{z}\left((k-1) t_{0}\right)=P_{k-1}, P_{z}\left(k t_{0}\right)=P_{k}\end{cases}
$$

Теоретические кривые, соответствующие модели № 6, для второй программы нагружения приведены на рис. 6.

3.3. Третья программа нагружения. Зависимости касательного $\tau$ и нормального $\sigma$ напряжений от времени $t$ в третьей программе приведены на рис. 3:

$$
\begin{array}{lll}
k=1,3,5,7,9: & \tau(t)=\frac{\sigma_{0}}{\sqrt{3}}, & \sigma(t)=0, \\
k=2,4,6,8,10: & \tau(t)=-\frac{\sigma_{0}}{2}, & \sigma(t)=\frac{\sigma_{0}}{2} .
\end{array}
$$

Из (1), (2) при учете (9) получаем следующие дифференциальные уравнения относительно характеристик тензора деформаций ползучести $p_{z}(t)$ и $\Gamma(t)$ :

$$
\begin{gathered}
\frac{d \Gamma}{d t}=\frac{D_{1}}{\left(p_{z}^{2}+\Gamma^{2}\right)^{\alpha / 2}} \text { при } \tau(t)=\frac{\sigma_{0}}{\sqrt{3}}, \frac{d \Gamma}{d t}=-\frac{\sqrt{3}}{2} \frac{D_{1}}{\left(p_{z}^{2}+\Gamma^{2}\right)^{\alpha / 2}} \text { при } \tau=-\frac{\sigma_{0}}{2} ; \\
\frac{d p_{z}}{d t}=\frac{D_{1}}{2\left(p_{z}^{2}+\Gamma^{2}\right)^{\alpha / 2}} \text { при } \sigma=\frac{\sigma_{0}}{2}, \frac{d p_{z}}{d t}=0 \text { при } \sigma=0 .
\end{gathered}
$$

В данной программе нагружения с помощью (10), (11) получаем следующие значения $\Gamma_{k}$ и $P_{k}$ :

1) если $k=1$, то $0<t<t_{0} ; \tau(t)=\frac{\sigma_{0}}{\sqrt{3}}, \sigma(t)=0, p_{z}(t)=0, P_{1}=0$; $\frac{d \Gamma}{d t}=\frac{D_{1}}{\Gamma^{\alpha}}, \int_{0}^{\Gamma_{1}} \Gamma^{\alpha} d \Gamma=D_{1} t_{0}, \Gamma_{1}=\Gamma\left(t_{0}\right)=\left[(\alpha+1) D_{1} t_{0}\right]^{\frac{1}{\alpha+1}} ;$ 

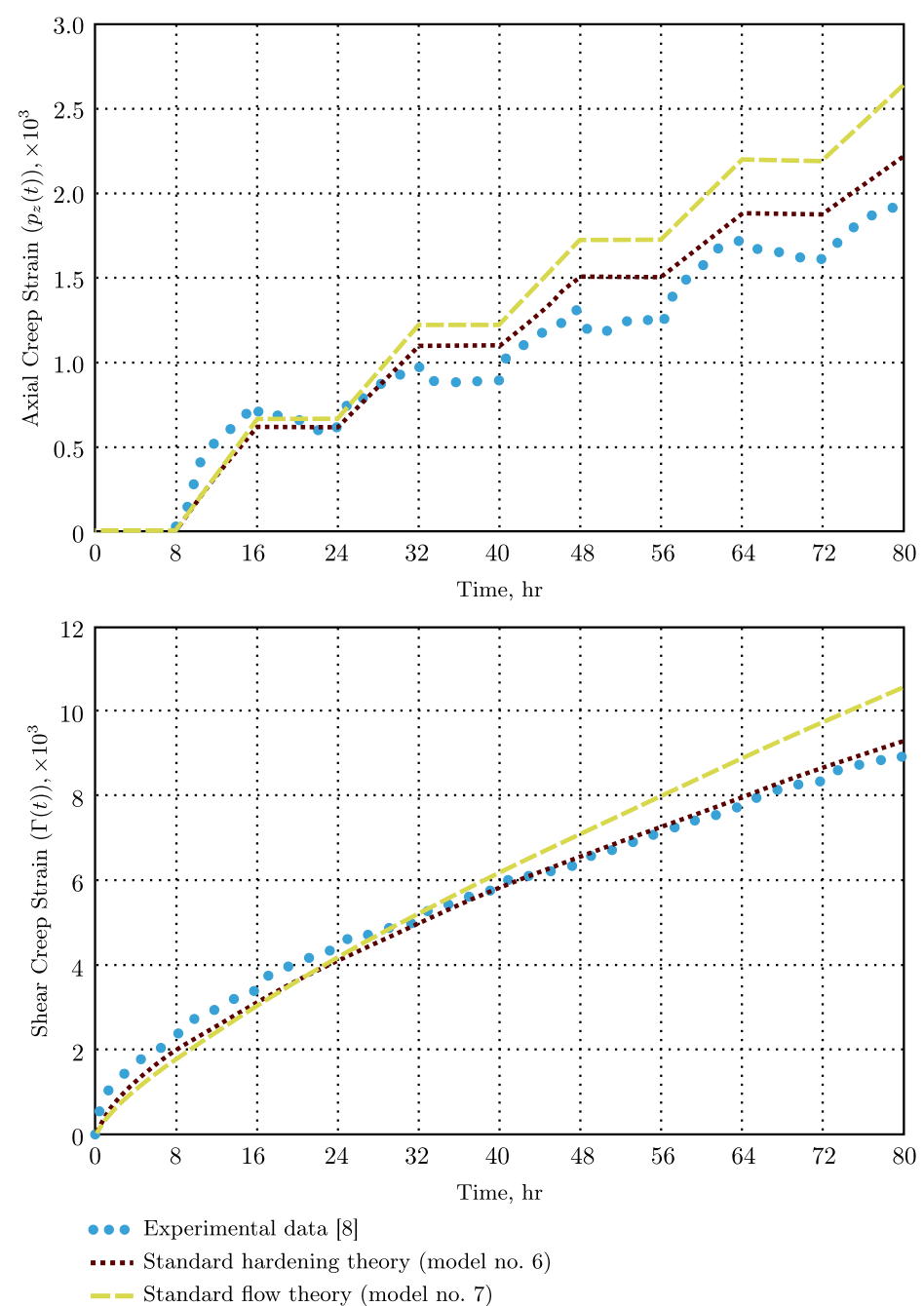

Рис. 6. (онлайн в цвете) Экспериментальные данные и результаты расчета по различным моделям для второй программы нагружения [Figure 6. (color online) Experimental data, and calculation results for various models for the second loading program]

2 ) если $k=2$, то $t_{0}<t<2 t_{0} ; \tau(t)=-\frac{\sigma_{0}}{2}, \sigma(t)=\frac{\sigma_{0}}{2}$;

$$
\begin{cases}\frac{d \Gamma}{d t}=-\frac{\sqrt{3}}{2} \frac{D_{1}}{\left(p_{z}^{2}+\Gamma^{2}\right)^{\alpha / 2}}, & \Gamma\left(t_{0}\right)=\Gamma_{1}, \quad \Gamma\left(2 t_{0}\right)=\Gamma_{2} \\ \frac{d p_{z}}{d t}=\frac{1}{2} \frac{D_{1}}{\left(p_{z}^{2}+\Gamma^{2}\right)^{\alpha / 2}}, & p_{z}\left(t_{0}\right)=P_{1}=0, \quad p_{z}\left(2 t_{0}\right)=P_{2}\end{cases}
$$

3) если $k=3,5,7,9$, то $(k-1) t_{0}<t<k t_{0} ; \tau(t)=-\frac{\sigma_{0}}{2}, \sigma(t)=0, P_{k}=P_{k=1}$; $\frac{d \Gamma}{d t}=\frac{D_{1}}{\left(P_{k}^{2}+\Gamma^{2}\right)^{\alpha / 2}}, \Gamma\left((k-1) t_{0}\right)=\Gamma_{k-1}, \Gamma\left(k t_{0}\right)=\Gamma_{k} ;$ 
4) если $k=4,6,8,10$, то $(k-1) t_{0}<t<k t_{0} ; \tau(t)=-\frac{\sigma_{0}}{2}, \sigma(t)=\frac{\sigma_{0}}{2}$;

$$
\begin{cases}\frac{d \Gamma}{d t}=-\frac{\sqrt{3}}{2} \frac{D_{1}}{\left(p_{z}^{2}+\Gamma^{2}\right)^{\alpha / 2}}, & \Gamma\left((k-1) t_{0}\right)=\Gamma_{k-1}, \quad \Gamma\left(k t_{0}\right)=\Gamma_{k} \\ \frac{d p_{z}}{d t}=\frac{1}{2} \frac{D_{1}}{\left(p_{z}^{2}+\Gamma^{2}\right)^{\alpha / 2}}, & p_{z}\left((k-1) t_{0}\right)=P_{k-1}, \quad p_{z}\left(k t_{0}\right)=P_{k} .\end{cases}
$$

Теоретические кривые, соответствующие модели № 6, для третьей программы нагружения приведены на рис. 7.
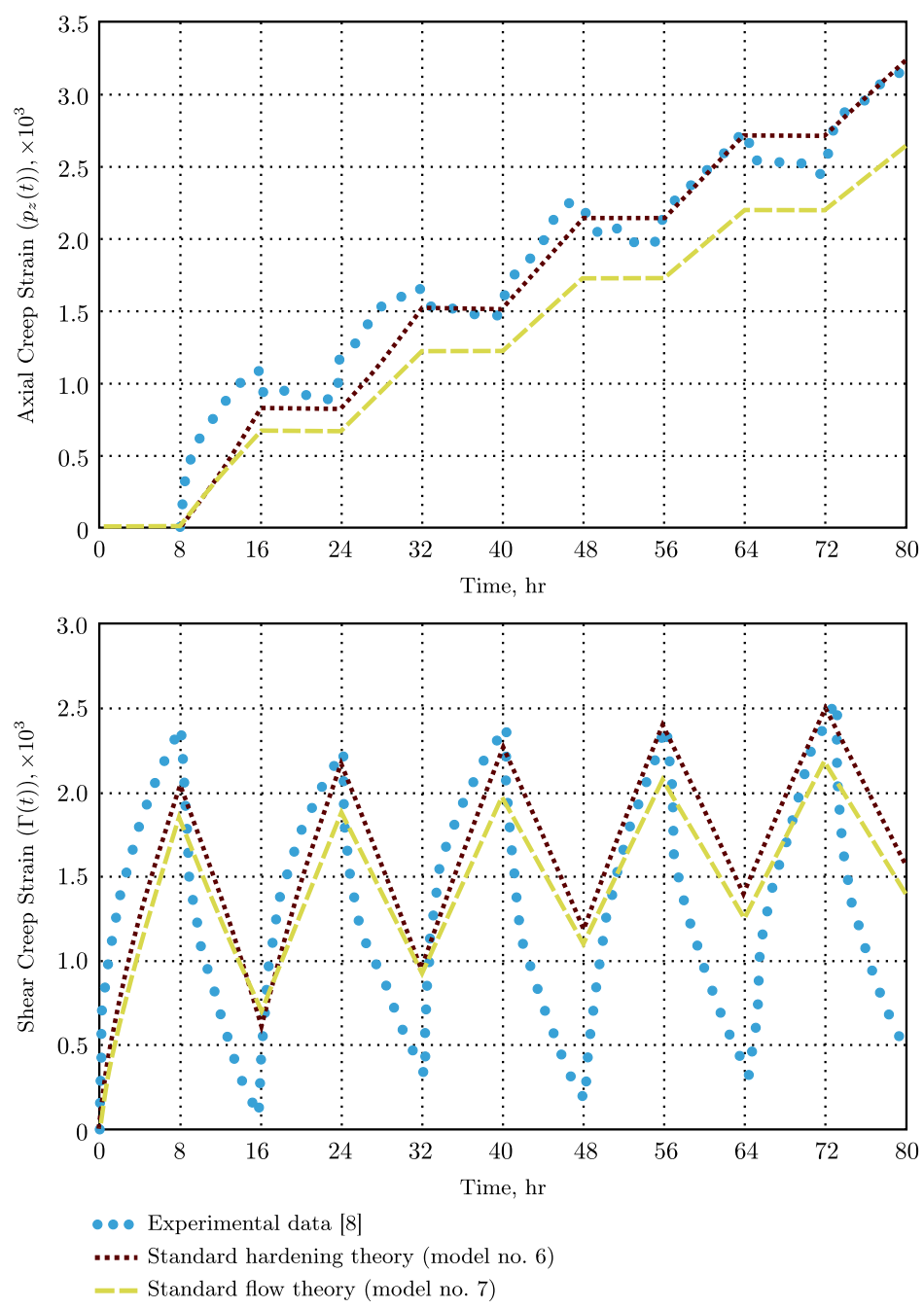

Рис. 7. (онлайн в цвете) Экспериментальные данные и результаты расчета по различным моделям для третьей программы нагружения [Figure 7. (color online) Experimental data, and calculation results for various models for the third loading program] 
3.4. Четвертая программа нагружения. В этой программе нагружения осуществлялось знакопеременное кручение, при этом нормальное напряжение отсутствовало (см. рис. 4):

$$
\tau(t)= \pm \frac{\sigma_{0}}{\sqrt{3}}, \quad \sigma(t)=0
$$

отсюда $p_{z}(t)=0$, т. е. $P_{1}=P_{2}=\cdots=P_{10}=0$. Использование $(2),(3)$ с учетом (12) приводит к следующим результатам:

1) если $k=1$, то $0<t<t_{0} ; \tau(t)=\frac{\sigma_{0}}{\sqrt{3}} ; \frac{d \Gamma}{d t}=\frac{D_{1}}{\Gamma^{\alpha}}, \Gamma(0)=0, \Gamma\left(t_{0}\right)=\Gamma_{1}$, $\int_{0}^{\Gamma_{1}} \Gamma^{\alpha} d \Gamma=\frac{\Gamma_{1}^{\alpha+1}}{\alpha+1}=D_{1} t_{0}, \Gamma_{1}=\left[(\alpha+1) D_{1} t_{0}\right]^{\frac{1}{\alpha+1}}$

$2)$ если $k=2$, то $t_{0}<t<2 t_{0} ; \tau(t)=-\frac{\sigma_{0}}{\sqrt{3}} ; \Gamma\left(t=2 t_{0}\right)=\Gamma_{2} ; \int_{\Gamma_{1}}^{\Gamma_{2}} \Gamma^{\alpha} d \Gamma=$ $=\frac{\Gamma_{2}^{\alpha+1}-\Gamma_{1}^{\alpha+1}}{\alpha+1}=-D_{1} t_{0} ; \Gamma_{2}^{\alpha+1}=\left[(\alpha+1) D_{1} t_{0}-(\alpha+1) D_{1} t_{0}\right]^{\frac{1}{\alpha+1}}=0 ;$ $\Gamma\left(2 t_{0}\right)=\Gamma_{2}=0$

3) проведя аналогичные вычисления при $k=3,4, \ldots, 10$, получим

$$
\Gamma_{1}=\Gamma_{3}=\Gamma_{5}=\Gamma_{7}=\Gamma_{9}=\left[(\alpha+1) D_{1} t_{0}\right]^{\frac{1}{\alpha+1}}, \quad \Gamma_{4}=\Gamma_{6}=\Gamma_{8}=\Gamma_{10}=0 .
$$

Теоретические кривые, соответствующие модели № 6 , для четвертой программы нагружения приведены на рис. 8.

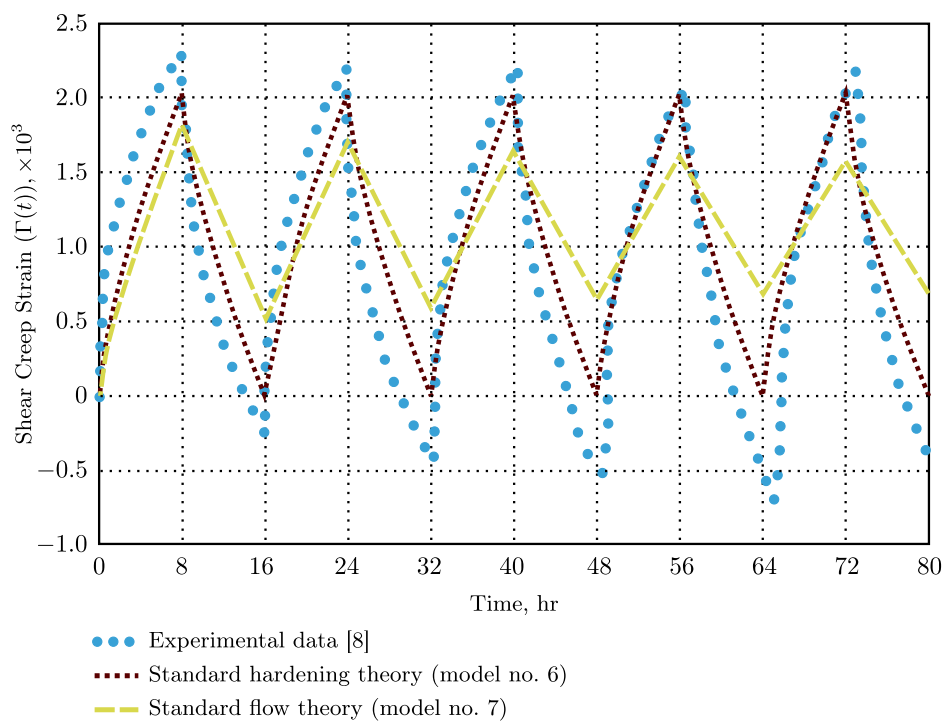

Рис. 8. (онлайн в цвете) Экспериментальные данные и результаты расчета по различным моделям для четвертой программы нагружения [Figure 8. (color online) Experimental data, and calculation results for various models for the fourth loading program] 
4. Теория течения (модель № 7). Рассмотрим теорию течения в следующем виде:

$$
\frac{d p_{u}}{d t}=\beta R \sigma_{0}^{m} t^{\beta-1}=\beta D_{2} t^{\beta-1}, \quad D_{2}=R \sigma_{0}^{m}, \quad 1<\beta<2 .
$$

По-прежнему будем использовать зависимости касательного $\tau(t)$ и нормального $\sigma(t)$ напряжений от времени $t$ (программы нагружения), представленные на рис. 1-4.

4.1. Первая программа нагружения. В этой программе нагружения компоненты тензора напряжений принимают значения (3). На основе уравнений (1), (2) получаем дифференциальные уравнения относительно осевых и касательных деформаций ползучести как функций времени:

$$
\begin{aligned}
& \frac{d \Gamma}{d t}=\beta D_{2} t^{\beta-1} \text { при } \tau(t)=\frac{\sigma_{0}}{\sqrt{3}}, \text { т. е. } d \Gamma=D_{2} d\left(t^{\beta}\right) ; \frac{d \Gamma}{d t}=0 \text { при } \tau(t)=0 ; \\
& \frac{p_{z}}{d t}=\beta D_{2} t^{\beta-1} \text { при } \sigma(t)=\sigma_{0}, \quad \text { т. е. } d p_{z}=D_{2} d\left(t^{\beta}\right) ; \frac{d p_{z}}{d t}=0 \text { при } \sigma(t)=0 .
\end{aligned}
$$

Далее имеем следующие результаты:

1) если $k=1$, то $0<t<t_{0} ; d \Gamma=R \sigma_{0}^{m} d\left(t^{\beta}\right), \Gamma\left(t_{0}\right)=\Gamma_{1}=D_{2} t_{0}^{\beta} ; P_{1}=$ $=p_{z}\left(t_{0}\right)=0$

$2)$ если $k=2$, то $t_{0}<t<2 t_{0} ; \Gamma\left(2 t_{0}\right)=\Gamma_{2}=\Gamma_{1} ; P_{2}=p_{z}\left(2 t_{0}\right)=D_{2}\left[\left(2 t_{0}\right)^{\beta}-\right.$ $\left.-t_{0}^{\beta}\right]+P_{1}$

$3)$ если $k=3,5,7,9$, то $(k-1) t_{0}<t<k t_{0} ; \Gamma_{k}=\Gamma\left(k t_{0}\right)=\Gamma_{k-1}+D_{2}\left[\left(k t_{0}\right)^{\beta}-\right.$ $\left.-\left((k-1) t_{0}\right)^{\beta}\right] ; P_{k}=P_{k-1}$

4) если $k=4,6,8,10$, то $(k-1) t_{0}<t<k t_{0} ; \Gamma_{k}=\Gamma_{k-1} ; P_{k}=P_{k-1}+$ $+D_{2}\left[\left(k t_{0}\right)^{\beta}-\left((k-1) t_{0}\right)^{\beta}\right]$.

Теоретические кривые, соответствующие модели № 7, для первой программы нагружения приведены на рис. 5.

4.2. Вторая программа нагружения. Эта программа нагружения характеризуется компонентами тензора напряжений (6). Уравнения (1), (2) при использовании (6) после преобразований приводят к следующим результатам:

$$
\begin{gathered}
d \Gamma=D_{2} d\left(t^{\beta}\right) \text { при } \tau=\frac{\sigma_{0}}{\sqrt{3}}, \quad d \Gamma=\frac{\sqrt{3}}{2} D_{2} d\left(t^{\beta}\right) \text { при } \tau=\frac{\sigma_{0}}{2} ; \\
d p_{z}=\frac{1}{2} D_{2} d\left(t^{\beta}\right) \text { при } \sigma=\frac{\sigma_{0}}{2}, \quad d p_{z}=0 \text { при } \sigma=0 .
\end{gathered}
$$

Далее имеем следующее:

1) если $k=1$, то $0<t<t_{0} ; \Gamma\left(t_{0}\right)=\Gamma_{1}=D_{2} t_{0}^{\beta} ; P_{1}=p_{z}\left(t_{0}\right)=0 ;$

2) если $k=2$, то $t_{0}<t<2 t_{0} ; \Gamma\left(2 t_{0}\right)=\Gamma_{2}=\Gamma_{1}+\frac{\sqrt{3}}{2} D_{2}\left[\left(2 t_{0}\right)^{\beta}-t_{0}^{\beta}\right]$;

$3)$ если $k=3,5,7,9$, то $\Gamma\left(k t_{0}\right)=\Gamma_{k}=\Gamma_{k-1}+D_{2}\left[\left(k t_{0}\right)^{\beta}-\left((k-1) t_{0}\right)^{\beta}\right]$; $p_{z}\left(k t_{0}\right)=P_{k}=P_{k-1}$

4) если $k=4,6,8,10$, то $\Gamma\left(k t_{0}\right)=\Gamma_{k}=\Gamma_{k-1}+\frac{\sqrt{3}}{2} D_{2}\left[\left(k t_{0}\right)^{\beta}-\left((k-1) t_{0}\right)^{\beta}\right]$; $p_{z}\left(k t_{0}\right)=P_{k}=P_{k-1}+\frac{1}{2} D_{2}\left[\left(k t_{0}\right)^{\beta}-\left((k-1) t_{0}\right)^{\beta}\right]$. 
Теоретические кривые, соответствующие модели № 7, для второй программы нагружения приведены на рис. 6.

4.3. Третья программа нагружения. Для третьей программы нагружения получаем, что

$$
\begin{gathered}
d \Gamma=D_{2} d\left(t^{\beta}\right) \text { при } \tau=\frac{\sigma_{0}}{\sqrt{3}}, \quad d \Gamma=-\frac{\sqrt{3}}{2} D_{2} d\left(t^{\beta}\right) \text { при } \tau=-\frac{\sigma_{0}}{2} ; \\
d p_{z}=\frac{1}{2} D_{2} \cdot d\left(t^{\beta}\right) \text { при } \sigma=\frac{\sigma_{0}}{2}, \quad d p_{z}=0 \text { при } \sigma=0 .
\end{gathered}
$$

Для каждой ступени нагружения получаем следующее:

1) если $k=1$, то $0<t<t_{0} ; \Gamma\left(t_{0}\right)=\Gamma_{1}=D_{2} t_{0}^{\beta} ; P_{1}=p_{z}\left(t_{0}\right)=0 ;$

2) если $k=2$, то $t_{0}<t<2 t_{0} ; \Gamma\left(2 t_{0}\right)=\Gamma_{2}=\Gamma_{1}-\frac{\sqrt{3}}{2} D_{2}\left[\left(2 t_{0}\right)^{\beta}-t_{0}^{\beta}\right]$;

$3)$ если $k=3,5,7,9$, то $(k-1) t_{0}<t<k t_{0} ; \Gamma\left(k t_{0}\right)=\Gamma_{k}=\Gamma_{k-1}+D_{2}\left[\left(k t_{0}\right)^{\beta}-\right.$ $\left.-\left((k-1) t_{0}\right)^{\beta}\right] ; P_{k}=P_{k-1}$

4) если $k=4,6,8,10$, то $(k-1) t_{0}<t<k t_{0} ; \Gamma_{k}=\Gamma_{k-1}-\frac{\sqrt{3}}{2} D_{2}\left[\left(k t_{0}\right)^{\beta}-\right.$ $\left.-\left((k-1) t_{0}\right)^{\beta}\right] ; P_{k}=P_{k-1}+\frac{1}{2} D_{2}\left[\left(k t_{0}\right)^{\beta}-\left((k-1) t_{0}\right)^{\beta}\right]$.

Теоретические кривые, соответствующие модели № 7, для третьей программы нагружения приведены на рис. 7.

4.4. Четвертая программа нагружения. Для четвертой программы имеем

$$
\begin{gathered}
d \Gamma=D_{2} d\left(t^{\beta}\right) \text { при } \tau=\frac{\sigma_{0}}{\sqrt{3}}, \quad d \Gamma=-D_{2} d\left(t^{\beta}\right) \text { при } \tau=-\frac{\sigma_{0}}{\sqrt{3}}, \\
d p_{z}=0 \text { при } \sigma=0 ; \text { следовательно, } P_{1}=P_{2}=P_{k}=0
\end{gathered}
$$

и аналогично получаем:

1) если $k=1$, то $0<t<t_{0} ; \Gamma\left(t_{0}\right)=\Gamma_{1}=D_{2} t_{0}^{\beta}$;

2) если $k=2$, то $t_{0}<t<2 t_{0} ; \Gamma\left(2 t_{0}\right)=\Gamma_{2}=\Gamma_{1}-D_{2}\left[\left(2 t_{0}\right)^{\beta}-t_{0}^{\beta}\right]$;

$3)$ если $k=3,5,7,9$, то $(k-1) t_{0}<t<k t_{0} ; \Gamma_{k}=\Gamma_{k-1}+D_{2}\left[\left(k t_{0}\right)^{\beta}-\right.$ $\left.-\left((k-1) t_{0}\right)^{\beta}\right]$

4) если $k=4,6,8,10$, то $(k-1) t_{0}<t<k t_{0} ; \Gamma_{k}=\Gamma_{k-1}-D_{2}\left[\left(k t_{0}\right)^{\beta}-\right.$ $\left.-\left((k-1) t_{0}\right)^{\beta}\right]$.

Теоретические кривые, соответствующие модели № 7 , для четвертой программы нагружения приведены на рис. 8.

5. Интегральный метод оценки погрешности при описании экспериментальных данных [8] различными моделями. В данном пункте предлагается интегральный способ оценки относительного расхождения экспериментальных величин деформаций ползучести (обозначены символом «звездочка») во всех четырех программах нагружения с теоретическими величинами, соответствующими различным моделям:

$$
\Delta_{\Gamma i}=\left(\int_{0}^{10 t_{0}}\left|\Gamma_{i}^{*}(t)-\Gamma_{i}(t)\right| d t\right) \cdot\left(\int_{0}^{10 t_{0}}\left|\Gamma_{i}^{*}(t)\right| d t\right)^{-1} \text { при } i=1,2,3,4 ;
$$




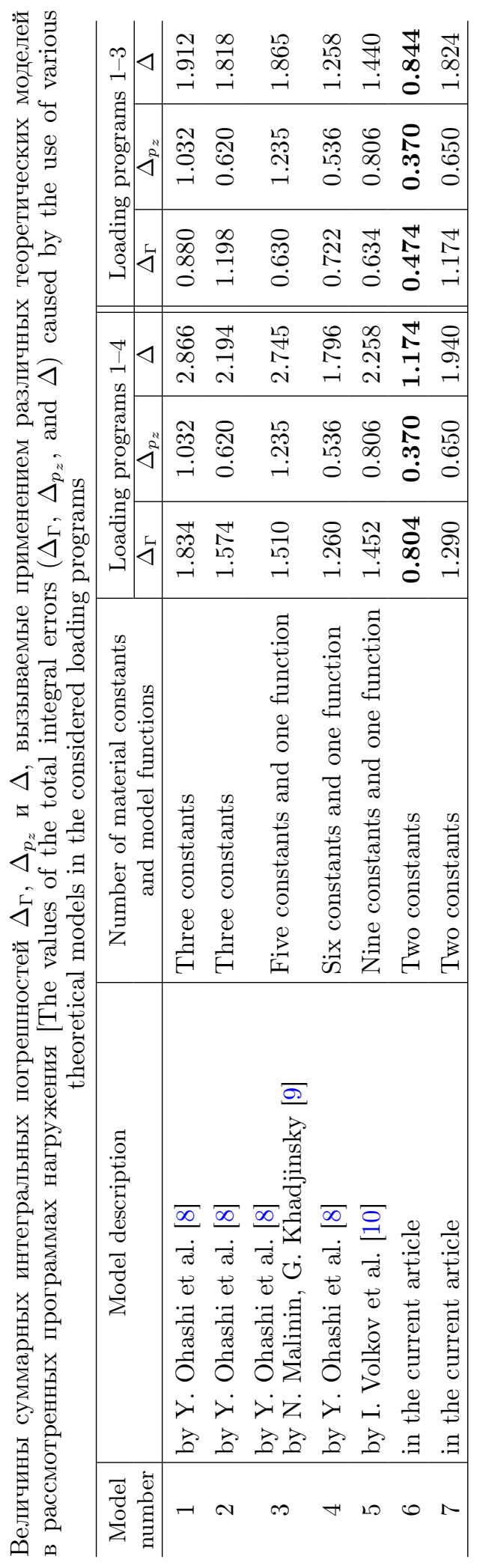




$$
\begin{gathered}
\Delta_{p_{z} i}=\left(\int_{0}^{10 t_{0}}\left|p_{z i}^{*}(t)-p_{z i}(t)\right| d t\right) \cdot\left(\int_{0}^{10 t_{0}}\left|p_{z i}^{*}(t)\right| d t\right)^{-1} \text { при } i=1,2,3, \\
\Delta_{p_{z} 4}=0 ; \\
\Delta_{\Gamma}=\sum_{i=1}^{4} \Delta_{\Gamma i}, \quad \Delta_{p_{z}}=\sum_{i=1}^{4} \Delta_{p_{z} i}, \quad \Delta=\Delta_{\Gamma}+\Delta_{p_{z}} .
\end{gathered}
$$

Значения $\Delta_{\Gamma}, \Delta_{p_{z}}$ и $\Delta$ для всех рассмотренных моделей приведены в таблице. При определении величин $\Delta_{\Gamma}, \Delta_{p_{z}}$ и $\Delta$ были использованы следующие значения материальных констант: $\alpha=0.46, D_{1}=8.0 \cdot 10^{-5}, \beta=0.786$, $D_{2}=0.00036$. Здесь необходимо отметить, что $D_{1}$ и $D_{2}$ являются постоянными для данной задачи, поскольку величина интенсивности напряжений $\sigma_{u}=\sigma_{0}=$ const является постоянной величиной на всех программах нагружения. Эти величины определены в результате оценки минимальных значений $\Delta$ соответственно для моделей № 6,7 . На рис. 5-8 приведены экспериментальные кривые ползучести и теоретические кривые, соответствующие моделям № 6,7 .

6. Обсуждение результатов и выводы. Из приведенных в таблице данных следует, что при описании одних и тех же экспериментальных зависимостей применение теории упрочнения всего с двумя материальными константами (модель № 6) приводит к значительно меньшей погрешности, чем применение моделей 1-5 со значительно большим количеством констант (до девяти материальных констант и одной материальной функции). Погрешности при применении теории течения (модель № 7) имеют тот же порядок, что и погрешности моделей $1-5$, хотя в ней использованы только две материальные константы.

Таким образом, авторами данной статьи показано преимущество рассмотренных теории упрочнения и теории течения всего с двумя материальными константами в каждой по сравнению с другими использованными теориями.

Конкурирующие интересы. Заявляем, что в отношении авторства и публикации этой статьи конфликта интересов не имеем.

Авторский вклад и ответственность. Все авторы принимали участие в разработке концепции статьи и в написании рукописи. Авторы несут полную ответственность за предоставление окончательной рукописи в печать. Окончательная версия рукописи была одобрена всеми авторами.

Финансирование. Работа выполнена при поддержке Российского научного фонда (РНФ 19-19-00062, Московский государственный университет имени М.В. Ломоносова).

Благодарность. Авторы благодарны рецензентам за тщательное прочтение статьи и ценные предложения и комментарии.

\section{Библиографический список}

1. Abo el Ata M. M., Finnie I. A study of creep damage rules // J. Basic Eng., 1972. vol. 94, no. 3. pp. 533-541. doi: 10.1115/1.3425474.

2. Trąmpczyński W. A., Hayhurst D. R., Leckie F. A. Creep rupture of copper and aluminum under non-proportional loading // J. Mech. Phys. Solids, 1981. vol. 29, no. 5-6. pp. 353-374.

3. Murakami S., Sanomura Y., Saitoh K. Formulation of cross-hardening in creep and its 
effect on the creep damage process of copper // J. Eng. Mater. Technol., 1986. vol. 108, no. 2. pp. 167-173. doi: 10.1115/1.3225856.

4. Trivaudey F., Delobelle P. High temperature creep damage under biaxial loading-Part I: Experiments // J. Eng. Mater. Technol., 1990. vol.112, no.4. pp. 442-449. doi: 10.1115/ 1.2903355.

5. Локощенко А. М. Применение векторного параметра поврежденности при моделировании длительной прочности металлов // Изв. РАН. МТT, 2016. № 3. С. 93-99.

6. Локощенко А. М. Моделирование длительной прочности металлов при нестационарном сложном напряженном состоянии // ПММ, 2018. Т. 82, № 1. С. 84-97.

7. Работнов Ю. Н. Ползучесть элементов конструкций. М.: Наука, 1966. 752 с.

8. Ohashi Y., Ohno N., Kawai M. Evaluation of creep constitutive equations for type 304 stainless steel under repeated multiaxial loadings // J. Eng. Mater. Technol., 1982. vol. 104, no. 3. pp. 159-164. doi: 10.1115/1.3225059.

9. Malinin N. N., Khadjinsky G. M. Theory of creep with anisotropic hardening // Int. J. Mech. Sciences, 1972. vol. 14, no. 4. pp. 235-246. doi: 10.1016/0020-7403(72)90065-3.

10. Волков И. А., Игумнов Л. А., Казаков Д. А., Шишулин Д. Н., Сметанин И. В. Определяющие соотношения нестационарной ползучести при сложном напряженном состоянии // Проблемы прочности и пластичности, 2016. Т.78, №4. С. 436-451. doi: $10.32326 / 1814-9146-2016-78-4-436-451$.

11. Волков И. А., Игумнов Л. А., Казаков Д. А., Шишулин Д. Н., Тарасов И. С. Уравнения состояния нестационарной ползучести при сложном нагружении // ПМТФ, 2018. № 3 . C. 191-202. doi : 10.15372/PMTF20180320.

12. Работнов Ю. Н. Расчет деталей машин на ползучесть// Изв. АН СССР. Отд. техн. наук, 1948. №6. С. 789-800.

13. Локощенко А. М. Ползучесть и длительная прочность металлов. М.: Физматлит, 2016. 504 c. 


\title{
MSC: 74A05, 74D10
}

\section{Simulation of metal creep in nonstationary complex stress state}

\author{
A. M. Lokoshchenko ${ }^{1}$, L. V. Fomin ${ }^{1}$, \\ Yu. G. Basalov ${ }^{1}$, V. S. Agababyan ${ }^{2}$ \\ 1 Lomonosov Moscow State University, \\ Institute of Mechanics, \\ 1, Michurinsky prospekt, Moscow, 119192, Russian Federation. \\ ${ }_{2}$ Lomonosov Moscow State University, \\ Faculty of Mechanics and Mathematics, \\ 1, Leninskie Gory, Moscow, 119991, Russian Federation.
}

\begin{abstract}
The simulation of the results of metal testing under creep conditions at the nonstationary complex stress state is considered. As an example, we consider the experimental data obtained by a group of Japanese scientists for testing tubular samples of stainless steel under the temperature of $650{ }^{\circ} \mathrm{C}$. The following article presents the test results for four different loading programs. These loading programs are various combinations of piecewise constant dependencies of tangential and normal stresses on time. The presented data was simulated using the hardening theory and the flow theory; two material constants used are determined from the condition of the minimum relative integral discrepancy between the experimental and theoretical values of the corresponding creep deformations. A comparison was made of the results of the simulation carried out with the results of the simulation of the same experimental data, carried out by other researchers using other theories. In these theories, a large number of material characteristics are used: from three to nine constants and additionally one material function. The advantage of the hardening theory and flow theory with only two material constants each compared to the other theories has been shown.
\end{abstract}

Keywords: metal creep, experiments, nonstationary complex stress state, modeling, hardening theory, flow theory.

\section{Research Article}

ㅇ (a) (1) The content is published under the terms of the Creative Commons Attribution 4.0 International License (http://creativecommons.org/licenses/by/4.0/)

Please cite this article in press as:

Lokoshchenko A. M., Fomin L. V., Basalov Yu. G., Agababyan V. S. Simulation of metal creep in nonstationary complex stress state, Vestn. Samar. Gos. Tekhn. Univ., Ser. Fiz.-Mat. Nauki [J. Samara State Tech. Univ., Ser. Phys. Math. Sci.], 2019, vol. 23, no. 1, pp. 69-89. doi: 10.14498/vsgtu1668 (In Russian).

\section{Authors' Details:}

Alexander M. Lokoshchenko (10) https://orcid.org/0000-0002-5462-6055

Dr. Phys. \& Math. Sci., Professor; Head of Laboratory; Lab. of Creep and Long-Term Strength; e-mail: loko@imec.msu.ru

Leonid V. Fomin (1) https://orcid.org/0000-0002-9075-5049

Cand. Phys. \& Math. Sci.; Senior Researcher; Lab. of Creep and Long-Term Strength; e-mail: fleonid1975@mail.ru

Yuriy G. Basalov; Lead Engineer; Lab. of Creep and Long-Term Strength; e-mail: basalov@yandex.ru

Vardan S. Agababyan; Student; Dept. of Gas and Wave Dynamics 
Received: $28^{\text {th }}$ December, 2018 / Revised: 26 ${ }^{\text {th }}$ February, $2019 /$

Accepted: $4^{\text {th }}$ March, $2019 /$ First online: $5^{\text {th }}$ March, 2019

Competing interests. We declare that we have no conflicts of interest in the authorship and publication of this article.

Authors' contributions and responsibilities. Each author has participated in the article concept development and in the manuscript writing. The authors are absolutely responsible for submitting the final manuscript in print. Each author has approved the final version of manuscript.

Funding. This study was supported by the Russian Science Foundation (RSF 19-1900062, Lomonosov Moscow State University).

Acknowledgments. The authors are grateful to the referees for careful reading of the paper and valuable suggestions and comments.

\section{References}

1. Abo el Ata M. M., Finnie I. A study of creep damage rules, J. Basic Eng., 1972, vol.94, no. 3, pp. 533-541. doi: 10.1115/1.3425474.

2. Trạmpczyński W. A., Hayhurst D. R., Leckie F. A. Creep rupture of copper and aluminum under non-proportional loading, J. Mech. Phys. Solids, 1981, vol. 29, no. 5-6, pp. 353-374.

3. Murakami S., Sanomura Y., Saitoh K. Formulation of cross-hardening in creep and its effect on the creep damage process of copper, J. Eng. Mater. Technol., 1986, vol. 108, no. 2, pp. 167-173. doi: 10.1115/1.3225856.

4. Trivaudey F., Delobelle P. High temperature creep damage under biaxial loading - Part I: Experiments, J. Eng. Mater. Technol., 1990, vol.112, no. 4, pp. 442-449. doi: 10.1115/1. 2903355.

5. Lokoshchenko A. M. Use of a vector damage parameter in modeling of long-term strength of metals, Mechanics of Solids, 2016, vol.51, no.3, pp. 315-320. doi: 10.3103/ S0025654416030080.

6. Lokoshchenko A. M. Modeling the long-term strength of metals in an unsteady complex stress state, Prikladnaya Matematika i Mekhanika, 2018, vol. 82, no. 1, pp. 84-97 (In Russian).

7. Rabotnov Yu. N. Creep problems in structural members. Amsterdam, London, NorthHolland Publ. Co., 1969, xiv+822 pp.

8. Ohashi Y., Ohno N., Kawai M. Evaluation of creep constitutive equations for type 304 stainless steel under repeated multiaxial loadings, J. Eng. Mater. Technol., 1982, vol. 104, no. 3, pp. 159-164. doi: 10.1115/1.3225059.

9. Malinin N. N., Khadjinsky G. M. Theory of creep with anisotropic hardening, Int. J. Mech. Sciences, 1972, vol. 14, no. 4, pp. 235-246. doi: 10.1016/0020-7403(72)90065-3.

10. Volkov I. A., Igumnov L. A., Kazakov D. A., Shishulin D. N., Smetanin I. V. Defining relations of transient creep under complex stress state, Problems of Strength and Plasticity, 2016, vol. 78, no. 4, pp. 436-451 (In Russian). doi: 10.32326/1814-9146-2016-78-4-436-451.

11. Volkov I. A., Igumnov L. A., Kazakov D. A., Shishulin D. N., Tarasov I. S. State equations of unsteady creep under complex loading, J. Appl. Mech. Tech. Phys., 2018, vol. 59, no. 3, pp. 551-560. doi: 10.1134/S0021894418030203.

12. Rabotnov Yu. N. Designing machine parts for creep, Izv. Akad. Nauk SSSR, OTN, 1948, no. 6, pp. 789-800 (In Russian).

13. Lokoshchenko A. M. Creep and long-term strength of metals. Boca, Raton, CRC Press, 2018, xviii+545 pp. doi : 10.1201/b22242. 